\title{
Genomic and transcriptomic analysis reveal molecular basis of salinity tolerance in a novel strong salt-tolerant rice landrace Changmaogu
}

Bing-Rui Sun ${ }^{1+}{ }^{\mathbb{D}}$, Chong-Yun Fu ${ }^{1+}$, Zhi-Lan Fan ${ }^{1,2}$, Yu Chen ${ }^{1,2}$, Wen-Feng Chen ${ }^{1,2}$, Jing Zhang ${ }^{1,2}$, Li-Qun Jiang ${ }^{1,2}$, Shuwei LV ${ }^{1,2}$, Da-Jian Pan ${ }^{1,2^{*}}$ and Chen $\mathrm{Li}^{1,2^{*}}$

\begin{abstract}
Background: Salt stress is an important factor that limits rice yield. We identified a novel, strongly salt tolerant rice landrace called Changmaogu (CMG) collected from a coastal beach of Zhanjiang, Guangdong Province, China. The salt tolerance of CMG was much better than that of the international recognized salt tolerant rice cultivar Pokkali in the germination and seedling stages.

Results: To understand the molecular basis of salt tolerance in CMG, we performed BSA-seq for two extreme bulks derived from the cross between CMG and a cultivar sensitive to salt, Zhefu802. Transcriptomic sequencing was conducted for CMG at the germination and young seedling stages. Six candidate regions for salt tolerance were mapped on Chromosome 1 by BSA-seq using the extreme populations. Based on the polymorphisms identified between both parents, we detected 32 genes containing nonsynonymous coding single nucleotide polymorphisms (SNPs) and frameshift mutations in the open reading frame (ORF) regions. With transcriptomic sequencing, we detected a large number of differentially expressed genes (DEGs) at the germination and seedling stages under salt stress. KEGG analysis indicated two of 69 DEGs shared at the germination and seedling stages were significantly enriched in the pathway of carotenoid biosynthesis. Of the 169 overlapping DEGs among three sample points at the seedling stage, 13 and six DEGs were clustered into the pathways of ABA signal transduction and carotenoid biosynthesis, respectively. Of the 32 genes carrying sequence variation, only OsPP2C8 (Os01g0656200) was differentially expressed in the young seedling stage under salt stress and also showed sequence polymorphism in the ORFs between CMG and Zhefu802.
\end{abstract}

Conclusion: OSPP2C8 was identified as the target candidate gene for salinity tolerance in the seedling stage. This provides an important genetic resource for the breeding of novel salt tolerant rice cultivars.

Keywords: Rice landrace, Salt tolerance, BSA-seq, Transcriptome sequencing

\footnotetext{
*Correspondence: 13922413514@163.com; lic11111@sina.com

${ }^{\dagger}$ Bing-Rui Sun and Chong-Yun Fu contributed equally to this work.

${ }^{1}$ Rice Research Institute, Guangdong Academy of Agricultural Sciences,

Guangzhou 510640, China

Full list of author information is available at the end of the article
} 
Salinity is one of the most common abiotic stresses limiting crop production. Except for in coastal areas, improper irrigation and the use of poor-quality water aggravate the salinization of arid and semi-arid soil in inland areas (Rahman et al., 2016). Rice (Oryza sativa L.) is a staple food crop that is salt sensitive in both young seedling and reproductive stages (Kumar et al., 2013). Identifying rice germplasm that is salt tolerant and breeding rice cultivars that are salt tolerant are the most economic and effective methods for the reduction of rice yield loss caused by salinity.

Rice salinity tolerance is a complex trait controlled by quantitative trait loci (QTLs) (Roy et al., 2011; Wang et al., 2013) and also shows different physiological mechanisms ( $\mathrm{Li}$ and $\mathrm{Xu}, 2007)$. Although many salt tolerant QTLs have been detected in different rice lines (Koyama et al., 2001; Ammar et al., 2009; Pandit et al., 2010; Gong et al., 1999; Kumar et al., 2015), only several major salt tolerant rice QTLs or genes such as qSKC1 (Lin et al., 2004; Ren et al., 2005), qSNC7 (Lin et al., 2004), Saltol (Thomson et al., 2010), and OsRR22 (Takagi et al., 2015) have been identified by genomic methods. A major QTL, qSKC1, encodes a sodium transporter in rice under salt stress (Ren et al., 2005). OsRR22 encodes a B-type response regulator protein that acts as a transcription factor regulating genes involved in osmotic responses and/or ion transport between parenchyma cells and vascular tissue cells of roots (Takagi et al., 2015).

Next generation sequencing (NGS) technologies contribute to discovering genome-wide genetic variation and genotyping in a highly efficient way (Huang and Han, 2013). The relatively low cost of sequencing enables the use of genome and transcriptomic sequencing to map some agronomic traits, especially quantitative traits (Varshney et al., 2014b; Pandey et al., 2017). The bulk segregant analysis (BSA) method is more effective in rapidly locating candidate genomic regions that underlie the target genes based on whole genomic resequencing for the extreme bulks and both parents (Takagi et al., 2013). Several transcriptomic studies have identified numerous differentially expressed genes in salinity-tolerant rice varieties compared to salinitysensitive rice varieties (Kawasaki et al., 2001; Walia et al., 2005; Cotsaftis et al., 2011; Wang et al., 2016; Shankar et al., 2016). These differentially expressed genes (DEGs) are generally associated with stress signaling, ion transport, transcription regulation, and some specific metabolic processes (Kawasaki et al., 2001; Walia et al., 2005; Cotsaftis et al., 2011; Kumar et al., 2013). However, current studies of the tolerance of rice to salinity mainly focus on the classic salt-tolerant rice cultivar, Pokkali, and its derived line, FL478. We identified a new salttolerant landrace called Changmaogu (CMG), which shows much stronger tolerance to salinity than Pokkali at the germination and young seedling stages (Fig. 1a).

In this study, we performed BSA sequencing for two extreme bulks and transcriptomic sequencing for CMG in the germination and young seedling stages under salt stress. This research aimed to identify the salt tolerant candidate genes in the seedling stage and provide insight into the molecular basis of salinity tolerance in CMG.

\section{Results}

Evaluation of Salinity Tolerance of CMG and Construction of the Extreme Mapping Population for BSA-Seq

The rice landrace Changmaogu (CMG), collected from a coastal beach of Zhanjiang, Guangdong Province, China, showed strong tolerance to the salinity of sea water (the total salt concentration: $370 \mathrm{mM}$ ) (Fig. 1a). To investigate the salt tolerance of CMG and identify the optimal salt concentration, the germination rates of CMG, Pokkali, and Zhefu802 were scored under six different salt $(\mathrm{NaCl})$ concentrations $(85 \mathrm{mM}, 100 \mathrm{mM}$, $120 \mathrm{mM}, 140 \mathrm{mM}, 150 \mathrm{mM}$, and $170 \mathrm{mM})$. The germination rates of the three rice lines gradually decreased with increasing salt concentration. Overall, CMG showed a much stronger tolerance to salinity than Pokkali and Zhefu802 under all six salt concentrations (Fig. 1b). The germination rate of CMG was similar to that of Pokkali and much greater than that of Zhefu802 in the $85 \mathrm{mM} \mathrm{NaCl}$ treatment. In the $100 \mathrm{mM} \mathrm{NaCl}$ treatment, the germination rate of CMG was higher than that of Pokkali. The germination rate of CMG varied based on the $\mathrm{NaCl}$ concentration; the germination rates were $69 \%, 48.67 \%$, and $18.7 \%$ in the 100,120 , and $140 \mathrm{mM} \mathrm{NaCl}$ treatments, respectively. Hence, the 120 $\mathrm{mM} \mathrm{NaCl}$ treatment was considered the optimal concentration. The germination rate of CMG, Pokkali, and Zhefu 802 were $48.67 \%, 17.33 \%$, and $4.67 \%$, respectively, in the $120 \mathrm{mM} \mathrm{NaCl}$ solution (Fig. 1b).

To map the genes related to salinity tolerance in CMG, we constructed an $F_{2}$ population derived from the cross between the strongly tolerant cultivar CMG and the sensitive cultivar Zhefu802. This $F_{2}$ population was used to identify the degree of salinity tolerance in the young seedling stage using $F_{2: 3}$ seeds in the $120 \mathrm{mM}$ $\mathrm{NaCl}$ treatment. We identified the salinity tolerance of more than $1000 \mathrm{~F}_{2: 3}$ seeds and detected 241 and $68 \mathrm{~F}_{2: 3}$ lines showing no germination and more than $40 \%$ germination rate, respectively. We continued to cultivate the $68 \mathrm{~F}_{2: 3}$ seeds for 25 days after salt treatment and selected 30 extremely tolerant individuals showing good growth for 25 days after salt stress and 30 individuals sensitive to salinity to construct the tolerant and sensitive salinity bulks for BSA-seq (Fig. 1c). 

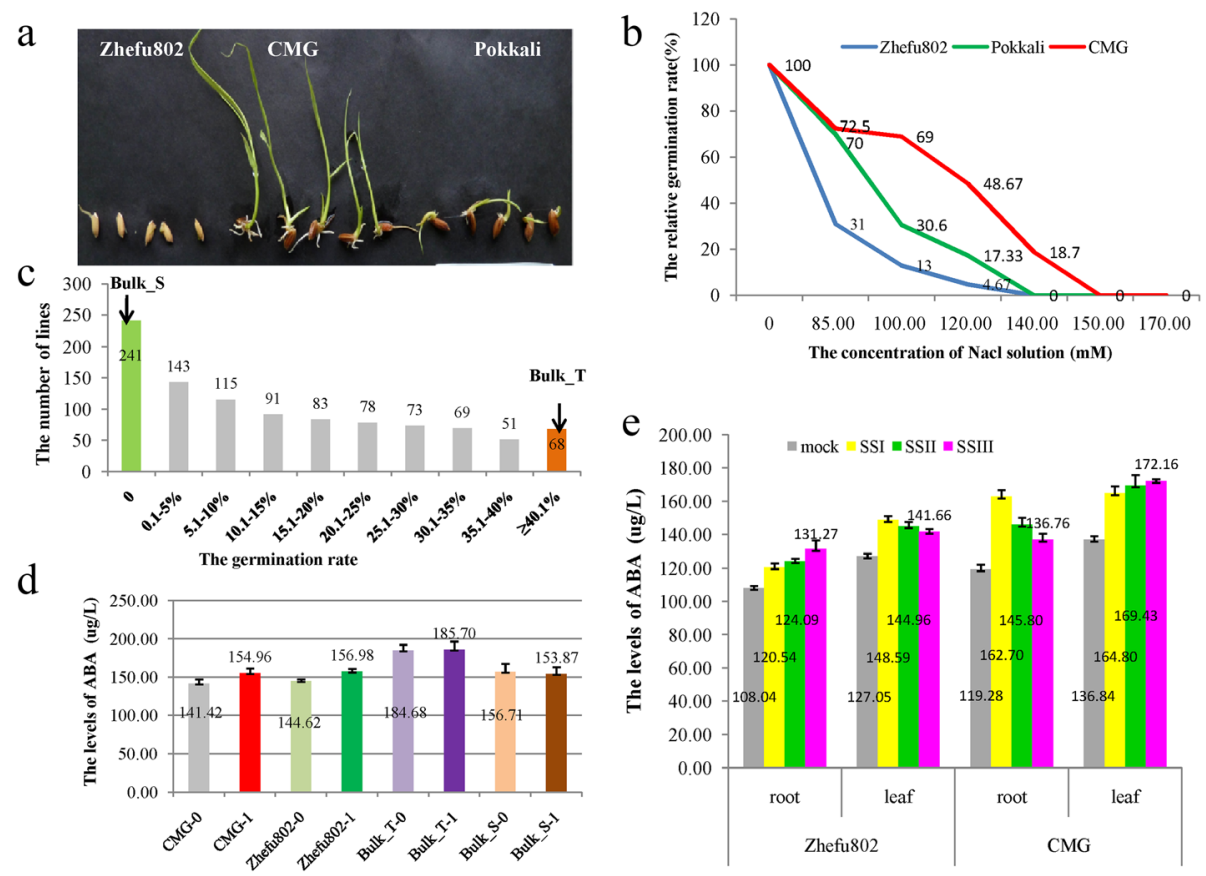

Fig. 1 The identification of salt tolerance and the assay of abscisic acid (ABA) in Zhefu802 and Changmaogu (CMG). a: The seedlings of CMG, Zhefu802, and Pokkali when the dry seeds were immersed in sea water (total salt:2\%) at room temperature for 30 days; b: The germination tendencies of CMG, Zhefu802, and Pokkali at different NaCl concentrations; $\mathbf{c}$ : The germination rates of CMG, Zhefu802, and Pokkali in 0.12 mol/L $\mathrm{NaCl}$ solution; $\mathbf{d}$ : the ABA levels of both parents and two bulks at germination stage; e: the ABA levels in roots and leaves of CMG at different sampling points under salt stress

\section{Measurement of Abscisic Acid (ABA)}

Abscisic acid (ABA) is a plant hormone that regulates plant growth and development and is rapidly increased by abiotic stresses such as drought and salinity (Mahajan and Tuteja, 2005). High salinity increases ABA (Kumar et al., 2013). To investigate whether the salt tolerance of CMG is associated with ABA, we tested the content of $\mathrm{ABA}$ in the roots and leaves under both normal growth conditions and salt stress at the germination and young seedling stage in CMG and Zhefu802. At the germination stage, the ABA levels in CMG and Zhefu802 increased under salt stress, compares with normal growth condition. But the ABA levels in CMG were equivalent to those in Zhefu802 under salt stress and normal growth condition, respectively. Compared with under normal growth condition, the ABA levels in both extreme bulks did not significantly change under salt stress. But the ABA levels (about $185 \mu \mathrm{g} / \mathrm{L}$ ) in extreme salt tolerant bulk were remarkably higher than those (about $154 \mu \mathrm{g} / \mathrm{L}$ ) in salt sensitive bulk under salt stress and normal growth condition (Fig. 1d). At the young seedling stage, compared with normal growth conditions, the ABA levels in roots and leaves increased under salt stress in both CMG and Zhefu802, and the increase of $\mathrm{ABA}$ in $\mathrm{CMG}$ was significantly higher than that in Zhefu802 (Fig. 1e). In roots of CMG, the ABA content instantly reached the highest level $(162.70 \mu \mathrm{g} / \mathrm{L})$ at the first sampling point (SSI) and then gradually reduced at SSII $(145.80 \mu \mathrm{g} / \mathrm{L})$ and SSIII $(136.76 \mu \mathrm{g} / \mathrm{L})$. In leaves of CMG, the ABA level gradually increased at three sampling points, SSI $(164.80 \mu \mathrm{g} / \mathrm{L})$, SSII $(169.43 \mu \mathrm{g} / \mathrm{L})$, and SSIII $(172.16 \mu \mathrm{g} / \mathrm{L})$.

However, Zhufu802 showed the different changing trend of $\mathrm{ABA}$ content in roots and leaves, compared with CMG. Namely, the ABA level gradually increased in roots $(120.54 \mu \mathrm{g} / \mathrm{L}, 124.09 \mu \mathrm{g} / \mathrm{L}$ and $131.27 \mu \mathrm{g} / \mathrm{L})$ and decreased in leaves $(148.59 \mu \mathrm{g} / \mathrm{L}, 144.96 \mu \mathrm{g} / \mathrm{L}$ and $141.66 \mu \mathrm{g} / \mathrm{L})$ at the sampling points from SSI to SSIII.

\section{Sequencing and Mapping of Reads to the 'Nipponbare' Reference Genome}

Whole genome re-sequencing data were generated for both parents and the extreme salinity tolerant bulk ( $\mathrm{T}_{-}$ bulk) and salinity sensitive bulk (S_bulk) (Table 1). A total of 42.15 million clean reads were generated for the salinity tolerant parent, CMG, 35.79 million clean reads for the salt sensitive parent, Zhefu802, 71.88 million clean reads for S_bulk, and 72.86 million clean reads for $\mathrm{T}$ bulk. The obtained sequencing data were $12.63 \mathrm{~Gb}$ for CMG, $10.72 \mathrm{~Gb}$ for Zhefu802, 21.54 Gbfor S_bulk, and $21.83 \mathrm{~Gb}$ for T_bulk, and more than $96 \%$ clean reads were mapped to the Nipponbare reference genome. The 
Table 1 Coverage of the reads mapping to the Nipponbare reference genome from resequencing of the indica rice variety Zhefu802 and a rice landrace CMG using Hiseq 2000.

\begin{tabular}{llllll}
\hline BMK ID & Clean_Reads & Clean_Base & Mapped (\%) & Properly_mapped (\%) & Q30(\%) \\
\hline CMG & $42,143,655$ & $12,625,063,756$ & 96.39 & 90.33 & 91.82 \\
Zhefu802 & $35,794,170$ & $10,720,972,860$ & 97.42 & 91.65 & 93.66 \\
S-pool & $71,884,976$ & $21,536,286,334$ & 98.01 & 91.94 \\
R-pool & $72,863,530$ & $21,826,349,910$ & 97.71 & 92.03 & 92.37 \\
\hline
\end{tabular}

rates of high quality (Q30) bases were more than 91\% (91.82\% for CMG, $93.66 \%$ for Zhefu802, 92.37\% for S_ bulk, and $92.78 \%$ for T_bulk).

\section{Candidate Regions for Salinity Tolerance by Bulked Segregant Analysis (BSA) Based on Genomic Resequencing}

In total, we detected 1,043,738 SNPs, including 96,226 nonsynonymous coding SNPs and 253,340 small InDels, between both parent lines. Between the two extreme bulks, we detected 331,923 SNPs including 32,239 nonsynonymous coding SNPs and 82,091 small InDels (Tables 2 and 3; Fig. 2a,b). An association analysis of salinity tolerance and polymorphic markers was performed using Euclidean Distance (ED) and SNP/InDel-index methods. We detected seven and six related candidate

Table 2 The distribution of detected SNPs between both parents Zhefu802 and CMG and between the two extreme pools

\begin{tabular}{lll}
\hline Type & Zhefu802_vs_CMG & S-pool_vs_R-pool \\
\hline Intergenic & 57,105 & 17,740 \\
Intragenic & 36 & 8 \\
Intron & 168,873 & 51,854 \\
Upstream & 345,387 & 109,660 \\
Downstream & 268,158 & 84,689 \\
UTR_5_Prime & 7979 & 2607 \\
UTR_3_Prime & 17,650 & 5114 \\
Splice_site_acceptor & 563 & 165 \\
Splice_site_donor & 542 & 165 \\
Splice_site_region & 3889 & 1224 \\
Start_gained & 1809 & 551 \\
Start_lost & 254 & 101 \\
Non_synonymous_start & 17 & 5 \\
Synonymous_coding & 69,741 & 24,034 \\
Non_synonymous_coding & 96,226 & 32,239 \\
Synonymous_stop & 97 & 34 \\
Stop_gained & 5114 & 1603 \\
Stop_lost & 298 & 128 \\
Other & 0 & 2 \\
Total & $1,043,738$ & 331,923 \\
\hline
\end{tabular}

regions covering $3.66 \mathrm{Mb}$ and $10.16 \mathrm{Mb}$ based on SNPindex and ED, respectively (Additional file 1: Table S1). Similarly, according to polymorphic InDels, we obtained 10 and nine candidate regions covering $3.63 \mathrm{Mb}$ and $9.58 \mathrm{Mb}$ based on InDel-index and ED, respectively (Additional file 1: Table S2). We overlapped the results based on the two methods, identifying six and six candidate regions covering $3.66 \mathrm{Mb}$ and $2.6 \mathrm{Mb}$, respectively, based on the threshold value of the confidence interval of 0.8914 of $\Delta(\mathrm{SNP} / \mathrm{InDel}$-index $)$ at the $95 \%$ significant level (Fig. 2c;Additional file 1: Table S3). Finally, with further analyses, we identified that the six overlapped regions as the most likely candidate regions for salinity tolerance at the seedling stage. These six candidate regions were located on chromosome 1 and covered a $2.6 \mathrm{Mb}$ region containing 430 genes (Table 4). Based on the sequence polymorphism in ORFs of 430 genes between CMG and Zhefu802, we found that 23 genes carried nonsynonymous coding SNPs and eight genes contained frame shift mutations (Table 5).

\section{Transcriptome Sequencing for CMG under Salt Stress at Germination and Seedling Stages}

To understand the differentially expressed genes responding to salt stress, we performed transcriptomic sequencing under the conditions of normal growth and salt stress (120 mM NaCl solution). We randomly selected 24 DEGs for real time PCR (RT-PCR) to validate the differential expression (Additional file 1: Table S4). The RT-PCR results were in good agreement with the transcriptomic sequencing results (Table 6). We investigated the number of upexpressed and downexpressed genes at germination and the seedling stage and found that the number of downregulated genes was greater than that of upregulated genes at the germination stage. The number of upregulated genes was greater than that of downregulated genes at the seedling stage, especially at the first sampling point (SSI) (Fig. 3a). Similarly, the number of differentially expressed transcription factors (TFs) had a similar tendency at the germination and seedling stages (Fig. 3b).

A Gene Ontology (GO) analysis was performed to explore the biological processes related to salinity tolerance at germination and seedling stages. At germination stage, the DEGs were significantly enriched in 48 biological 
Table 3 The distribution of detected InDels between both parents Zhefu802 and CMG and between the two extreme bulks

\begin{tabular}{|c|c|c|}
\hline Type & Zhefu802_vs_CMG & S-bulk_vs_R-bulk \\
\hline Intergenic & 14,431 & 4650 \\
\hline Intragenic & 126 & 46 \\
\hline Intron & 46,092 & 14,549 \\
\hline Upstream & 92,827 & 30,240 \\
\hline Downstream & 71,637 & 22,681 \\
\hline Utr_5_prime & 5138 & 1906 \\
\hline Utr_3_prime & 6685 & 2067 \\
\hline Splice_site_acceptor & 123 & 42 \\
\hline Splice_site_donor & 163 & 51 \\
\hline Splice_site_region & 816 & 261 \\
\hline Start_lost & 70 & 26 \\
\hline Frame_shift & 8918 & 3219 \\
\hline Codon_deletion & 1952 & 719 \\
\hline Codon_insertion & 2147 & 829 \\
\hline Exon_deleted & 1 & 0 \\
\hline Codon_change_plus_codon_insertion & 636 & 251 \\
\hline Codon_change_plus_codon_deletion & 1280 & 430 \\
\hline Stop_gained & 224 & 86 \\
\hline Stop_lost & 74 & 37 \\
\hline Other & 0 & 1 \\
\hline Total & 253,340 & 82,091 \\
\hline
\end{tabular}

process terms (FDR $<0.001)$, including oxidationreduction process (GO:0055114)(276 genes), carotenoid biosynthetic process (GO:0016117) (73 genes), glucosinolate biosynthetic process (GO:0019761) (71 genes), glutathione biosynthetic process (GO: 0006750) (71 DEGs), and response to karrikin (GO:0080167) (87 genes). The upregulated DEGs were significantly enriched into 21 terms (FDR < 0.001) (Fig. 3c), including glutathione metabolic process (GO:0006749), cadmium ion transport (GO:0015691), oxidation-reduction process (GO:0055114), negative regulation of response to water deprivation (GO:0080148), and proline transport (GO: 0015824). The downregulated DEGs were clustered into $46 \mathrm{GO}$ terms $(\mathrm{FDR}<0.001)$ (Fig. 3d). Most of these enriched terms were associated with the processes of photosynthesis and catalytic activity.

At the first sampling point (SSI) in the seedling stage, the DEGs were grouped into $21 \mathrm{GO}$ terms $(\mathrm{FDR}<0.001)$ (Fig. 4a), including glutathione metabolic process (GO: 0006749) (18 genes), response to karrikin (GO:0080167) (62 genes), response to water deprivation (GO:0009414) (111 genes), oxidation-reduction process (GO:0055114) (146 genes), negative regulation of response to water deprivation (GO:0080148) (14 genes), negative regulation of abscisic acid-activated signaling pathway (GO: 0009788) (23 genes), and proline transport (GO:
0015824) (26 genes). At SSII, these DEGs were clustered into nine GO terms (FDR < 0.001) containing response to water deprivation (GO:0009414) (161 genes), response to jasmonic acid (GO:0009753) (89 genes), and response to karrikin (GO:0080167) (73 genes) (Fig. 4b). At SSIII, the DEGs were also enriched into nine GO terms (FDR < 0.001) (Fig. 4c), including response to water deprivation (GO:0009414) (89 genes), response to water (GO: 0009415) (seven genes), response to desiccation (GO: 0009269) (24 genes) and oxidation-reduction process (GO:0055114) (112 genes).

We also investigated the overlapping DEGs between germination and seedling stages and among the three sampling points at the seedling stage. Some of the 69 shared DEGs between germination and the seedling stage were enriched in six GO terms (FDR < 0.01) (Fig. 5a), such as proline transport (GO:0015824) (seven genes), glutathione metabolic process (GO:0006749) (four genes), and negative regulation of response to water deprivation (GO:0080148) (three genes). The KEGG analysis indicated that only two genes, Os03g0645900 (OsNCED3) and Os03g0645966, were significantly enriched in the carotenoid biosynthesis pathway (ko00906) (FDR < 0.01) (Fig. 5b). The 164 overlapping genes among the three sampling points at the seedling stage were clustered into $13 \mathrm{GO}$ terms (FDR < 0.001) (Fig. $5 \mathrm{c})$, including negative regulation of abscisic acid-activated 


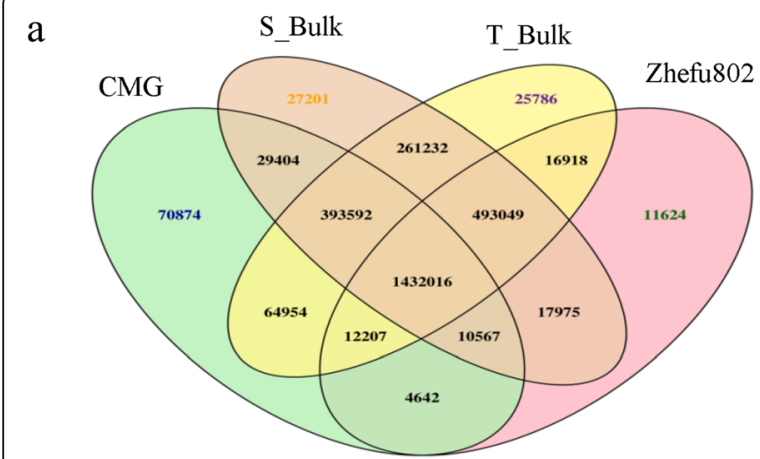

$\mathrm{b}$

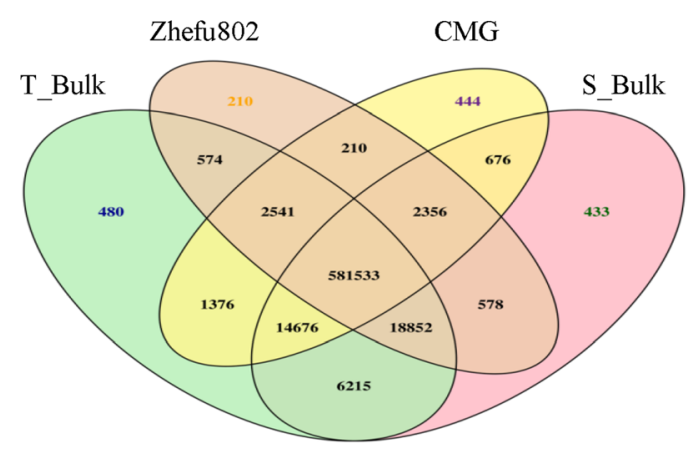

$\mathrm{c}$
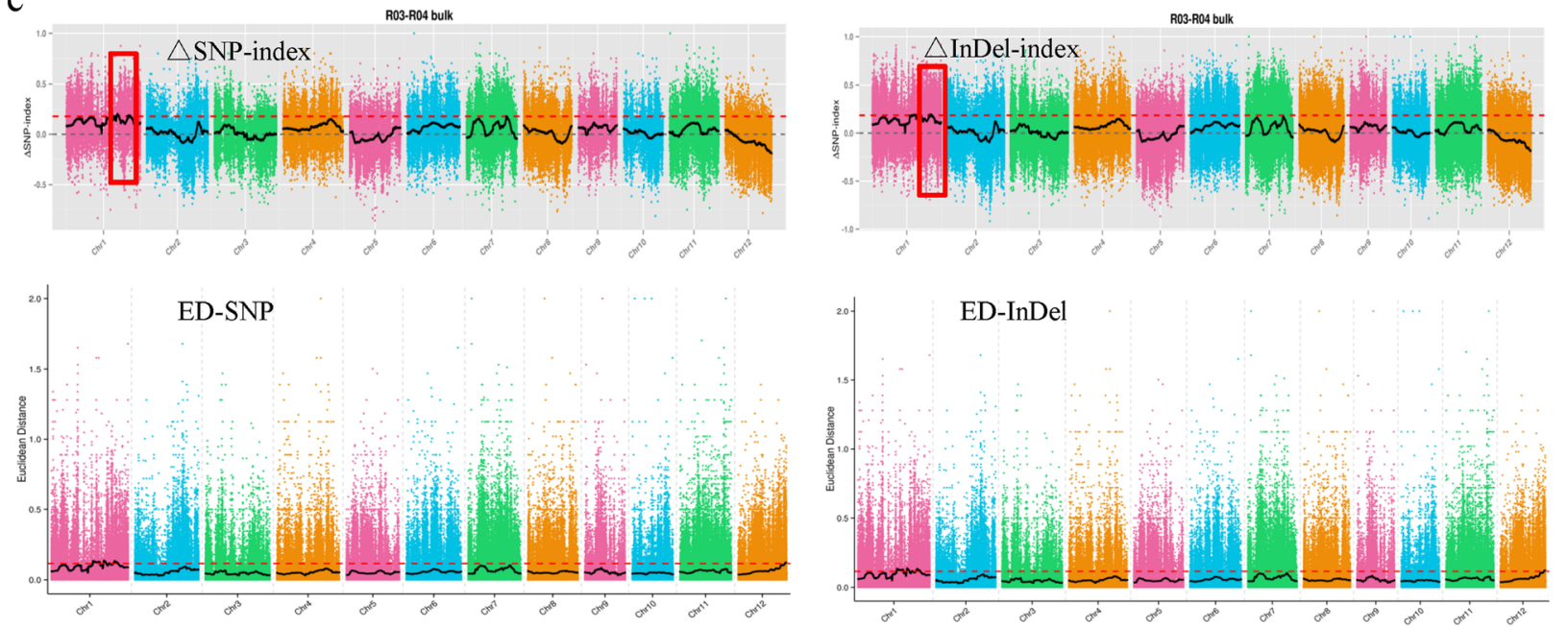

Fig. 2 Constructing the extremely sensitive (S_bulk) and tolerant (T_bulk) salt bulks and BSA-seq analysis for both parents, CMG and Zhefu802, and the two extreme bulks. a: The frequency distribution of the germination rate in more than $1000 \mathrm{~F}_{2: 3}$ lines from CMG and Zhefu802; $\mathbf{b}$ : Venn diagram of the number of SNPs in CMG, Zhefu802, S_bulk, and T_bulk; c: Venn diagram of the number of small InDels in CMG, Zhefu802, S_bulk, and T_bulk; $\mathbf{d}$ : the distribution of associated values based on SNP-/InDel-index and Euclidean Distance (ED) on different chromosomes

signaling pathway (GO:0009788) (13 genes), response to water deprivation (GO:0009414) (24 genes), hyperosmotic salinity response (GO:0042538) (16 genes), response to water (GO:0009415) (four genes), and regulation of stomatal movement (GO:0010119) (11 genes). These DEGs were also significantly grouped into the pathways of plant

Table 4 The detailed positions of the candidate regions for salt tolerance in CMG

\begin{tabular}{llll}
\hline Chromosome & Start & End & Size (Mb) \\
\hline Chr.1 & $26,110,000$ & $26,130,000$ & 0.020001 \\
Chr.1 & $26,160,000$ & $26,180,000$ & 0.020001 \\
Chr.1 & $26,490,000$ & $27,300,000$ & 0.810001 \\
Chr.1 & $29,440,000$ & $30,630,000$ & 1.190001 \\
Chr.1 & $35,800,000$ & $35,940,000$ & 0.140001 \\
Chr.1 & $36,140,000$ & $36,160,000$ & 0.020001 \\
Total & - & - & 2.200006 \\
\hline
\end{tabular}

hormone signal transduction (ko04075, 13 genes) and carotenoid biosynthesis (ko00906, six genes) (FDR $<0.00001$ ) (Fig. 5d). All of the 13 DEGs enriched for plant hormone signaling pathways are involved in abscisic acid (ABA) signal transduction (Fig. 5d). Of them, two DEGs (Os03g0297600 and Os05g0473101) belongs to the PYR1/ PYL family, 10 genes (Os01g0656200, Os01g0656250, Os01g0846150, Os01g0846300, Os03g0268600, Os03g026 8750, Os05g0457200, Os05g0457300, Os05g0537400, and Os09g0325700) belong to the protein phosphatase $2 \mathrm{C}$ family, and one gene (Os02g0766700) encodes a bZIP transcription factor acting as an ABA responsive element binding factor.

\section{Expression Patterns of Shared Genes among Three Sampling Points in the Seedling Stage}

We further analyzed the expression patterns of DEGs among three sampling points at the early seedling stage according to four expression patterns: Up-Up-Up (U-UU), Down-Down-Down (D-D-D), Down-Up-Down (D- 
Table 5 The candidate genes for salt tolerance based on the index and ED of SNP and InDel

\begin{tabular}{|c|c|c|c|}
\hline Mutation type & Gene ID & Position & Fuctional annotation \\
\hline \multirow[t]{23}{*}{ nonsynonymous coding SNP } & Os01g0655400 & $26,608,476-26,601,904$ & Transposon protein, putative \\
\hline & Os01g0733200 & $30,582,485-30,583,743$ & HSF-type DNA-binding domain containing protein \\
\hline & Os01g0656200 & $26,652,944-26,658,313$ & Protein phosphatase $2 \mathrm{C}$, putative, expressed \\
\hline & Os01g0655500 & $26,611,799-26,618,704$ & Serine/threonine-protein kinase stt7 \\
\hline & Os01g0647800 & $26,114,190-26,111,942$ & Hypothetical protein \\
\hline & Os01g0655600 & $26,619,462-26,620,234$ & Hypothetical protein \\
\hline & Os01g0729800 & $30,422,055-30,422,387$ & Hypothetical protein \\
\hline & Os01g0658300 & $26,769,190-26,767,671$ & Microneme protein Sm70 putative, expressed \\
\hline & Os01g0715100 & $30,516,831-30,517,430$ & Ubiquitin-related 4 (Precursor) \\
\hline & Os01g0715100 & $29,740,123-29,742,527$ & Conserved hypothetical protein \\
\hline & Os01g0733100 & $30,585,423-30,585,100$ & Hypothetical protein \\
\hline & Os01g0655700 & $26,622,829-26,624,411$ & Hypothetical protein \\
\hline & Os01g0656600 & $26,706,158-26,699,189$ & Hypothetical protein \\
\hline & Os01g0665500 & $27,200,990-27,196,417$ & Probable WRKY transcription factor 71 \\
\hline & Os01g0714700 & $29,717,779-29,718,346$ & Hypothetical protein \\
\hline & Os01g0659900 & $26,872,217-26,875,033$ & F-box domain and kelch repeat containing protein \\
\hline & Os01g0727600 & $30,341,594-30,340,015$ & Conserved hypothetical protein \\
\hline & Os01g0712250 & $29,590,343-29,589,249$ & Arginine/serine-rich protein \\
\hline & Os01g0722100 & $30,102,785-30,107,361$ & Bacterial transferase hexapeptide domain containing protein \\
\hline & Os01g0732100 & $30,533,818-30,536,908$ & Hypothetical protein \\
\hline & Os01g0655250 & $26,592,688-26,593,702$ & PWWP domain containing protein \\
\hline & Os01g0655300 & $26,599,136-26,599,632$ & Similar to Trithorax 4 \\
\hline & Os01g0719150 & $29,971,297-29,971,882$ & Hypothetical protein \\
\hline \multirow[t]{8}{*}{ Frameshift mutation } & Os01g0733200 & $30,582,485-30,583,743$ & HSF-type DNA-binding domain containing protein \\
\hline & Os01g0659400 & $26,823,851-26,822,203$ & Non-protein coding transcript \\
\hline & Os01g0655700 & $26,622,829-26,624,411$ & Hypothetical protein \\
\hline & Os01g0655600 & $26,619,462-26,620,234$ & Hypothetical protein \\
\hline & Os01g0733100 & $30,585,423-30,585,100$ & Cortical cell-delineating protein (Precursor) \\
\hline & Os01g0712250 & $29,590,343-29,589,249$ & Arginine/serine-rich protein 45 \\
\hline & Os01g0655250 & $26,592,688-26,593,702$ & PWWP domain containing protein \\
\hline & Os01g0655300 & $26,599,136-26,599,632$ & Similar to Trithorax 4 \\
\hline
\end{tabular}

U-D), and Up-Down-Up (U-D-U). The number of DEGs in the expression patterns U-U-U, D-D-D, D-U-D, and U-D-U were 92, 10, 16, and 29, respectively (Fig. 6a, Additional file 1: TableS5). The DEGs in the expression patterns D-U-D and U-D-U were not significantly enriched in any KEGG pathway. Some DEGs in the expression pattern U-U-U were grouped into the two pathways of plant hormone signal transduction (ko04075) (11 genes: Os01g0656200, Os01g0656250, Os01g084615 0, Os01g0846300, Os03g0268600, Os03g0268750, Os05g 0457200, Os05g0457300, Os05g0537400, Os09g0325700, and Os02g0766700) and carotenoid biosynthesis (ko00906) (six genes: Os09g0555500, Os03g0125100, Os03g0645900, Os03g0645966, Os07g0154100, and
Os07g0154201) (Fig. 5d, marked in red). Two DEGs (Os03g0297600 and Os05g0473101) in the expression pattern D-D-D were also clustered into the plant hormone signal transduction pathway (Fig. 5d, marked in green).

We also investigated the transcription factors (TFs) involved in the four expression patterns. We detected 10 TFs including two ABA-inducible bHLH-type TFs (Os01g0705700 and Os01g0705750), two C-2b heat shock TFs (Os06g0553001 and Os06g0553100), one ethyleneresponsive TF RAP2-3 (Os05g0361700), one GAMYB TF (Os04g0508500), one MYB23 TF (Os02g0618400), one NAC TF ONAC010 (Os03g0327800), one probable WRKY TF 57 (newGene_197), one HEC2 TF (Os09g0455300), 
Table 6 The expression value of 24 randomly selected genes in transcriptomic sequencing and RT-PCR

\begin{tabular}{|c|c|c|c|c|c|c|c|c|}
\hline \multirow[t]{2}{*}{ Gene ID } & \multicolumn{4}{|c|}{ Transcriptomic sequencing (FPKM) } & \multicolumn{4}{|c|}{ RT-PCR } \\
\hline & Mock & SSI & SSII & SSIII & Mock & SSI & SSIII & SSIII \\
\hline Os01g0135700 & 8.68 & 21.53 & 26.66 & 29.28 & 1.00 & 3.04 & 3.58 & 3.87 \\
\hline Os01g0656200 & 2.21 & 6.82 & 8.56 & 4.98 & 1.00 & 2.86 & 3.21 & 2.33 \\
\hline Os01g0699400 & 2.13 & 20.09 & 20.49 & 25.22 & 1.00 & 8.97 & 8.69 & 9.54 \\
\hline Os01g0705700 & 0.15 & 0.98 & 1.67 & 4.13 & 1.00 & 4.31 & 6.74 & 9.24 \\
\hline Os01g0756300 & 8.88 & 16.91 & 34.55 & 18.67 & 1.00 & 2.13 & 5.57 & 4.31 \\
\hline Os01g0846300 & 16.04 & 54.68 & 135.19 & 72.51 & 1.00 & 2.98 & 7.64 & 7.52 \\
\hline Os02g0179600 & 1.65 & 3.71 & 7.33 & 14.93 & 1.00 & 2.16 & 3.68 & 5.96 \\
\hline Os02g0618400 & 4.77 & 21.75 & 6.26 & 10.57 & 1.00 & 3.86 & 2.31 & 2.67 \\
\hline Os02g0682300 & 27.73 & 111.55 & 43.08 & 53.33 & 1.00 & 4.25 & 1.98 & 2.69 \\
\hline Os02g0766700 & 21.46 & 44.27 & 138.76 & 52.40 & 1.00 & 2.23 & 4.39 & 2.28 \\
\hline Os03g0197100 & 3.50 & 6.48 & 34.52 & 10.20 & 1.00 & 1.98 & 5.68 & 2.54 \\
\hline Os03g0327800 & 22.15 & 65.80 & 51.06 & 39.94 & 1.00 & 3.57 & 2.11 & 4.65 \\
\hline Os04g0508500 & 1.92 & 8.16 & 48.37 & 5.11 & 1.00 & 5.64 & 10.85 & 8.65 \\
\hline Os04g0585050 & 15.28 & 50.67 & 14.57 & 22.81 & 1.00 & 2.38 & 1.64 & 1.36 \\
\hline Os05g0361700 & 10.28 & 21.08 & 85.17 & 25.56 & 1.00 & 2.26 & 5.39 & 4.57 \\
\hline Os05g0381400 & 1.30 & 11.28 & 59.61 & 44.86 & 1.00 & 6.31 & 42.68 & 38.05 \\
\hline Os05g0457200 & 0.21 & 1.04 & 10.28 & 7.35 & 1.00 & 2.98 & 15.37 & 6.92 \\
\hline Os06g0553100 & 2.62 & 13.43 & 23.85 & 8.42 & 1.00 & 3.36 & 8.65 & 5.65 \\
\hline Os09g0332300 & 31.55 & 67.81 & 32.45 & 22.78 & 1.00 & 2.11 & 1.02 & 0.76 \\
\hline Os09g0455300 & 1.59 & 4.85 & 19.77 & 4.57 & 1.00 & 2.38 & 5.78 & 5.64 \\
\hline Os01g0908600 & 11.46 & 4.93 & 1.55 & 6.53 & 1.00 & 0.55 & 0.12 & 0.74 \\
\hline Os03g0297600 & 78.65 & 24.70 & 11.37 & 31.74 & 1.00 & 0.32 & 0.36 & 0.44 \\
\hline Os10g0552800 & 9.99 & 3.85 & 3.06 & 4.48 & 1.00 & 0.31 & 0.26 & 0.22 \\
\hline Os01g0655500 & 14.26 & 17.89 & 22.52 & 27.83 & 1.00 & 1.59 & 1.64 & 1.91 \\
\hline
\end{tabular}

and 1 bZIP TF BZIP23 (Os02g0766700) (Fig. 6d). All of these TFs were significantly up-regulated at three sampling points in the seedling stage.

\section{Screening the Target Candidate Genes for Salinity Tolerance}

In 31 possible candidate genes including 23 genes containing nonsynonymous coding SNPs and eight frameshift coding genes (Table 5), nonsynonymous and frameshift mutations simultaneously occurred in Os01g0712250 and Os01g0655700. One gene (Os01g0655400) encodes a putative transposon protein and 13 genes encode hypothetical proteins. We investigated the expression of the remaining 17 genes using transcriptomic sequencing. Only Os01g0656200 (OsPP2C8), encoding a protein phosphatase $2 \mathrm{C}$ family protein $(\mathrm{PP} 2 \mathrm{C})$, had significantly increased expression levels at the young seedling stage; the expression levels were not altered at the germination stage under the salt treatment. Compared with normal growth conditions, the expression of other genes did not change in the salt treatment. We also compared the sequence of
Os01g0656200 (OsPP2C8) in CMG and Zhefu802 according to BSA sequencing, and we detected one SNP (A in Zhefu802 to G in CMG) in the CDS region of OsPP2C8causing the alternation of the corresponding amino acid (Thr in Zhefu802 to Ala in CMG) (Fig. 6a). We also developed the corresponding primers for the variant site and validated the variant site by sequencing and aligning PCR products from CMG (Fig. 6b). Hence, OsPP2C8 was thought to be the most likely candidate gene for conferring salinity tolerance in the seedling stage in CMG.

\section{Discussion}

Rice is considered relatively tolerant to salt at germination and sensitive to salinity at the young seedling and reproductive stages (Heenanet al., 1988; Khatun et al., 1995; Zeng et al., 2001). Rice salinity tolerance is also thought to be a quantitative trait. Conventional QTL analysis is a laborious and time-consuming process because of the requirement of genotyping and phenotyping a large number of individuals derived from a biparental cross (Lim et al., 2014). Whole genomic sequencing 


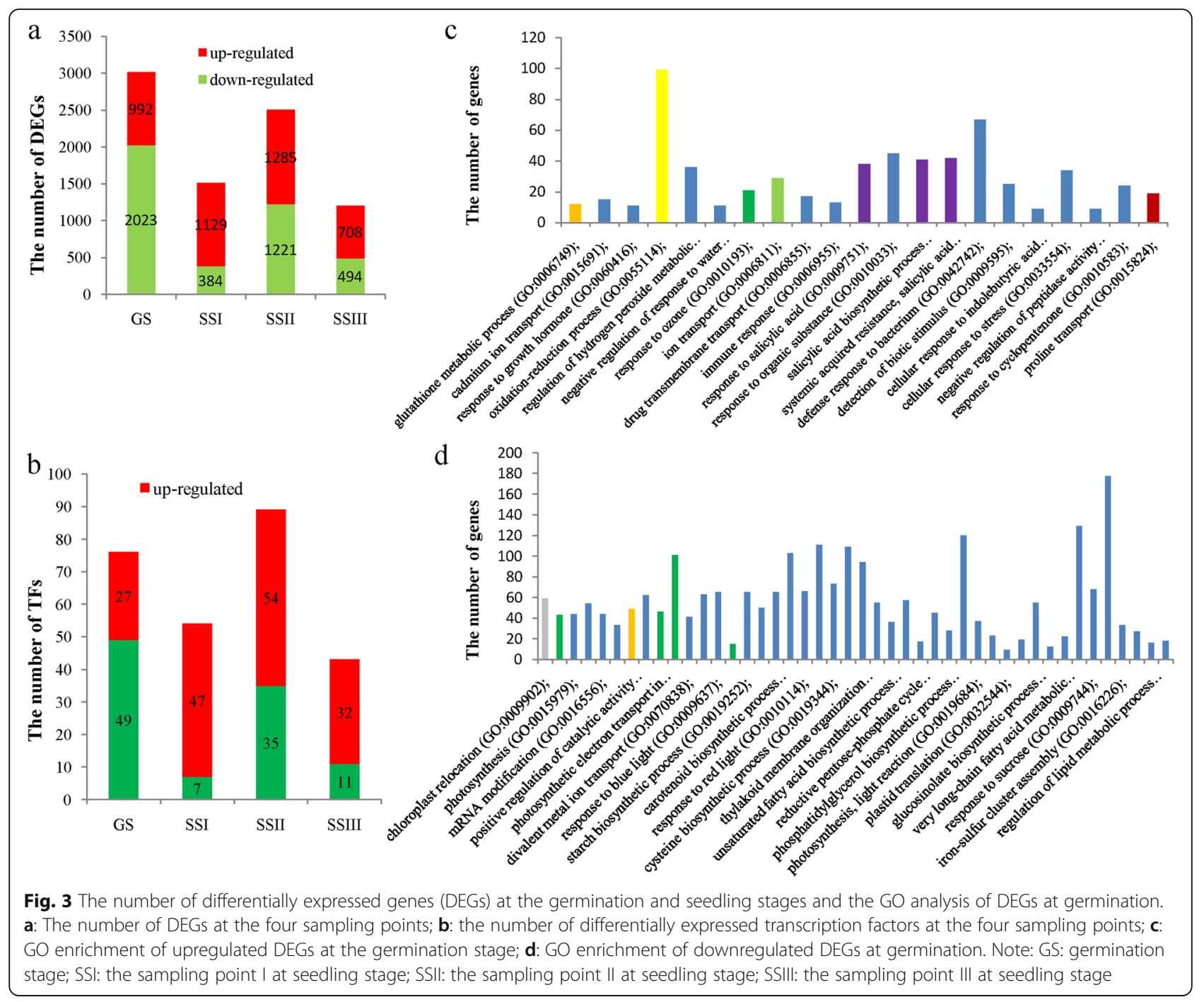

assisted BSA has been widely used for the analysis of quantitative traits controlled by a few major genes and is thought to be less useful for the identification of minor QTLs (Guo et al., 2017). In this study, we performed a BSA analysis based on whole genome sequencing and transcriptome sequencing of rice subjected to salt stress to identify the major QTLs for salinity tolerance in the rice landrace CMG, which has a strong tolerance to salinity.

Mapping Candidate Regions for Salinity Tolerance in CMG In the whole genome sequencing analysis, we detected a large number of SNPs and InDelInDels between CMG and Zhufu802 and obtained six candidate regions for salinity tolerance in CMG. In these six regions, three regions are small (approximately $20 \mathrm{~kb}$; Chr1: 26110000$26,130,000,26,160,000-26,180,000$, and 36,140,000-36, $160,000)$ and contained two to three putative genes, respectively. Os01g0648000 (located in the region from $26,110,000-26,130,000$ on Chr1) encodes a putative potassium channel protein that has been previously shown to be associated with salt stress (Fuchs et al., 2005); however, it was not differentially expressed in our transcriptase analysis.

Another two candidate regions are large $(0.81 \mathrm{Mb}$ spanning 26,490,000-27300000and $1.19 \mathrm{Mb}$ from 29, $440,000-30,630,000$ on Chr1). In the candidate region from 26,490,000-27,300,000, we detected a sequence variant in the CDS region of Os01g0656200, the expression of which was upregulated in the seedling stage under the salt stress treatment. In agreement with these results, a previous study also showed that Os01g0656200 was associated with salt tolerance in different salt tolerant rice lines (Walia et al., 2005; Pandit et al., 2010). Os01g0656200 (OsPP2C8) encodes a type of protein phosphatase $2 \mathrm{C}$ family protein $(\mathrm{PP} 2 \mathrm{C})$. $\mathrm{PP} 2 \mathrm{C}$ was known 


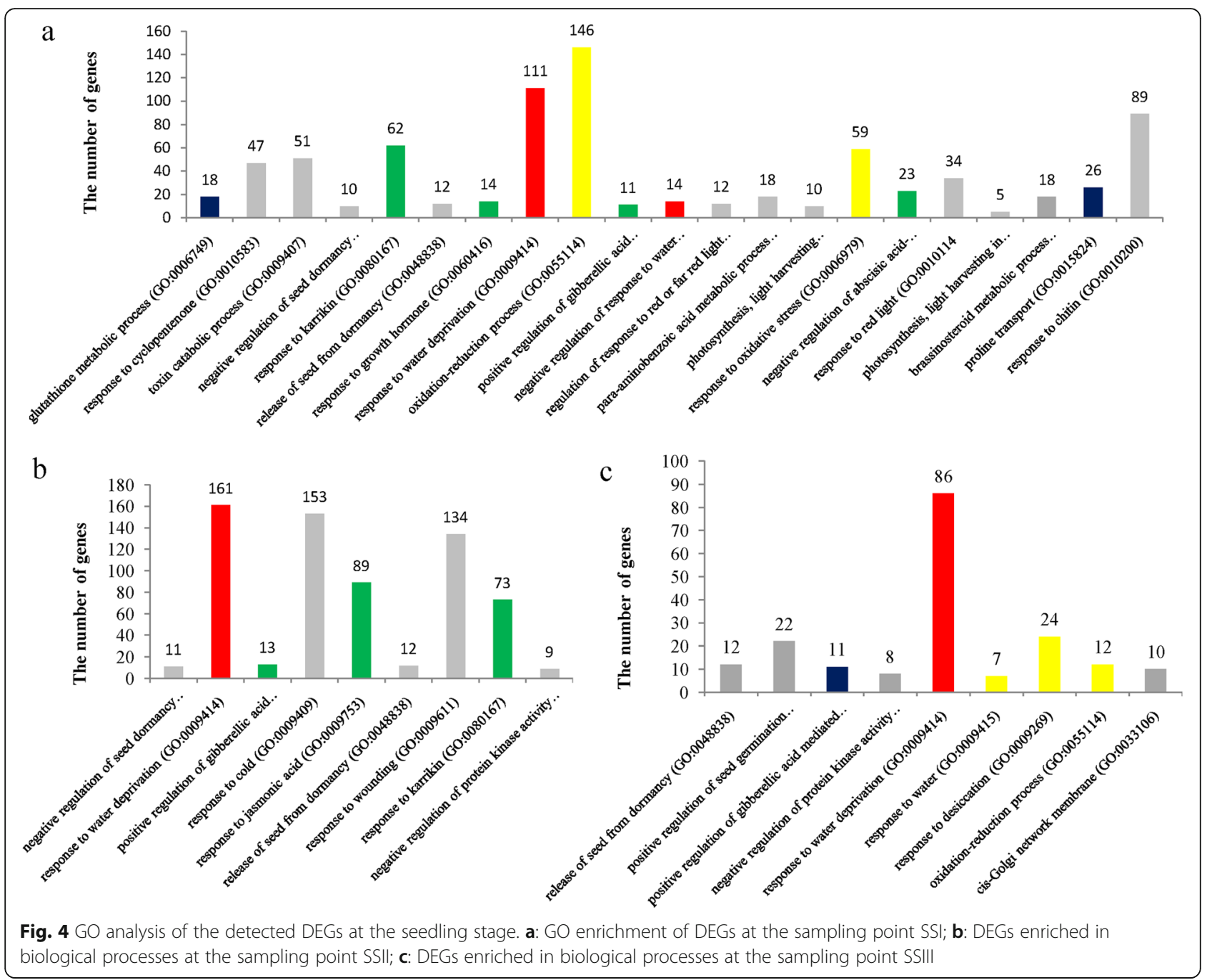

to be involved in abscisic acid signal transduction in higher plants (Sheen, 1998). Three PP2Cs (AtPP2CG1, AtPP2CA, and OsPP2C51) were found to enhance salinity tolerance in an abscisic acid-dependent mannerin Arabidopsis and promoted seed germination in rice (Liu et al., 2012; Cui et al., 2013; Bhatnagar et al., 2017). We detected a high level of ABA in the leaves and roots of CMG under salt stress in the seedling stage and the expression of OsPP2C8 was significantly up-regulated at the three sampling points in the seedling stage. CMG shows strong salinity tolerance in the germination and seedling stages, and the expression of this gene was significantly up-regulated in the seedling stages under salt stress. Hence, OsPP2C8 is also considered the most likely candidate gene for salt tolerance in CMG.

\section{Plant Hormone and Salinity Tolerance}

Plant hormones are thought to be the most important endogenous substances for regulating various physiological responses that lead to adaptation to salinity (Pearce et al., 2001b). Yang et al. (2014) assayed the levels of gibberellin, cytokinin, auxin, and abscisic acid under salt stress in tomato, and the study indicated that ABA played a major role in tomato salt tolerance. The exogenous application of ABA was found to offset the effects of osmotic and ion stress from salt stress conditions in commonbean (Khadri et al., 2007), wheat (Gurmani et al., 2013), and rice (Sripinyowanich et al., 2013) by reducing the sodium concentration and improving osmotic adjustment. In this study, we also found that the ABA level largely increased in the leaves and roots, even after approximately $30 \mathrm{~min}$ under salinity stress. Overlapping DEGs among three seedling sampling points were enriched in the pathways of carotenoid biosynthesis and ABA signal transduction, and two DEGs shared in the germination and seedling stages were also clustered into carotenoid biosynthesis. Duan et al. (2012) reported that the genes responsive to salt stress in the tomato line 'Moneymaker' and its wild genotype 


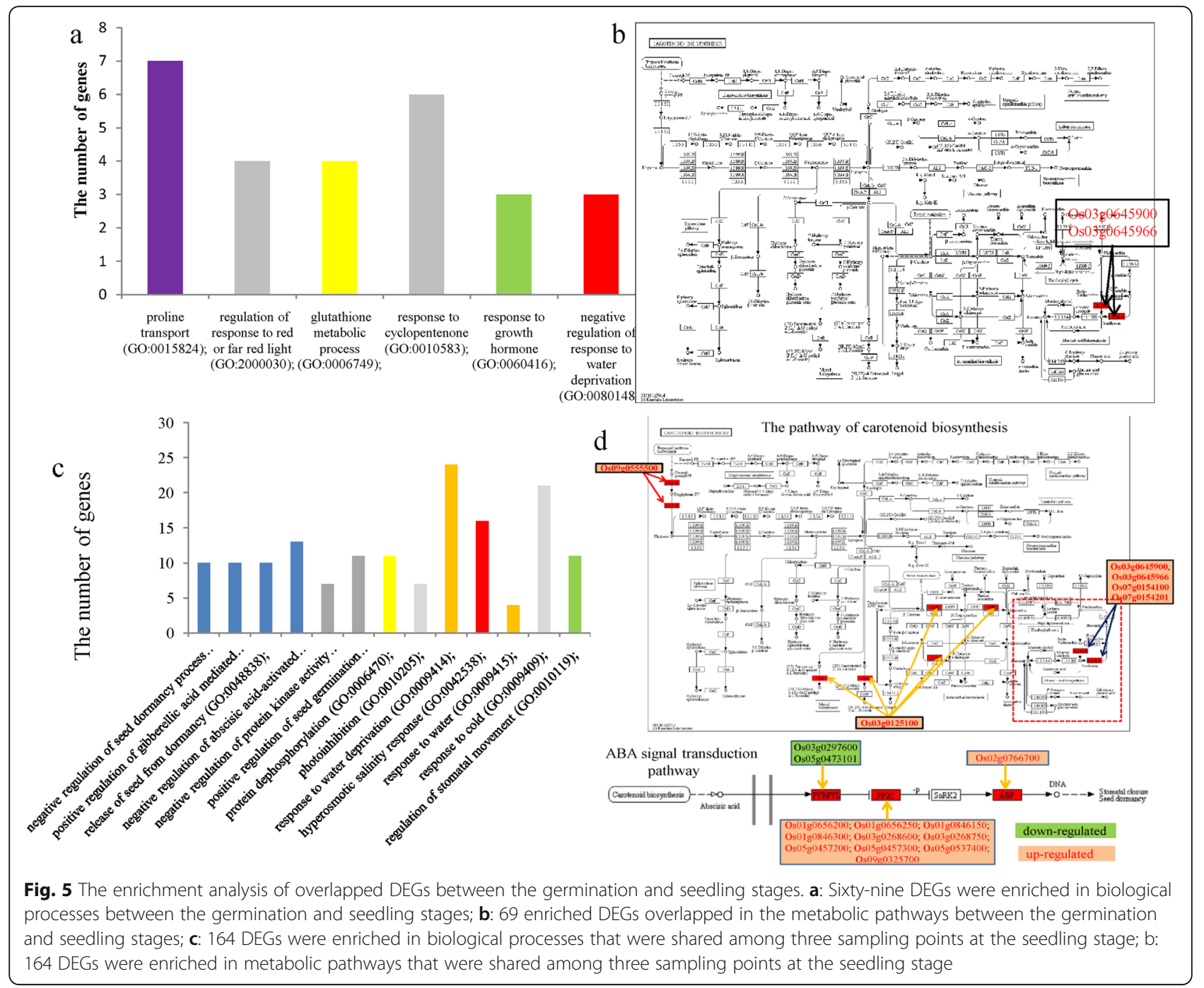

PI365967 (tolerant to salt) were involved in the carotenoid and ABA biosynthetic pathway. Similar results were also obtained in a close relative of Arabidopsis, Thellungiella, which showed strong tolerance to salt (Wong et al., 2006).

Combined with the above studies, these results likely imply that similar mechanisms in the adaptation to salinity stress apply for both cultivated plants and wild species, namely, rapid accumulation of ABA upon salinity stress condition set off the ABA-dependent signal transduction pathway to activate downstream target genes to respond to salt stress.

The transcriptome analysis under salt stress indicated that only two genes OsNCED3 (Os03g0645900) and Os03g0645966, a hypothetical gene of 69 DEGs were significantly clustered into the pathway of carotenoid biosynthesis. Interestingly, OsNCED3 was down-expressed in the germination stage and upexpressed at three sampling points in the seedling stage. Overexpression of rice OsNCED3 increased the accumulation of ABA, reduced relative water loss, and delayed seed germination in Arabidopsis (Hwang et al., 2010). In the sequence variants of the two genes in the BSA-seq analysis, we found that there were three nonsynonymous SNPs in the CDS regions. It is possible that down-expression of OsNCED3 decreases the level of ABA to promote seed germination in CMG in the germination stage. In addition, no shared DEGs among the germination and seedling stages were identified in plant hormone signaling pathways, which seems to indicate that the mechanism of salinity tolerance in the germination stage might be different from that in the seedling stage. Hence, OsNCED3 could be invovled into salinity tolerance in CMG at the germination stage, but it was not mapped into the candidate region based on the mapping population of CMG/Zhefu802. 


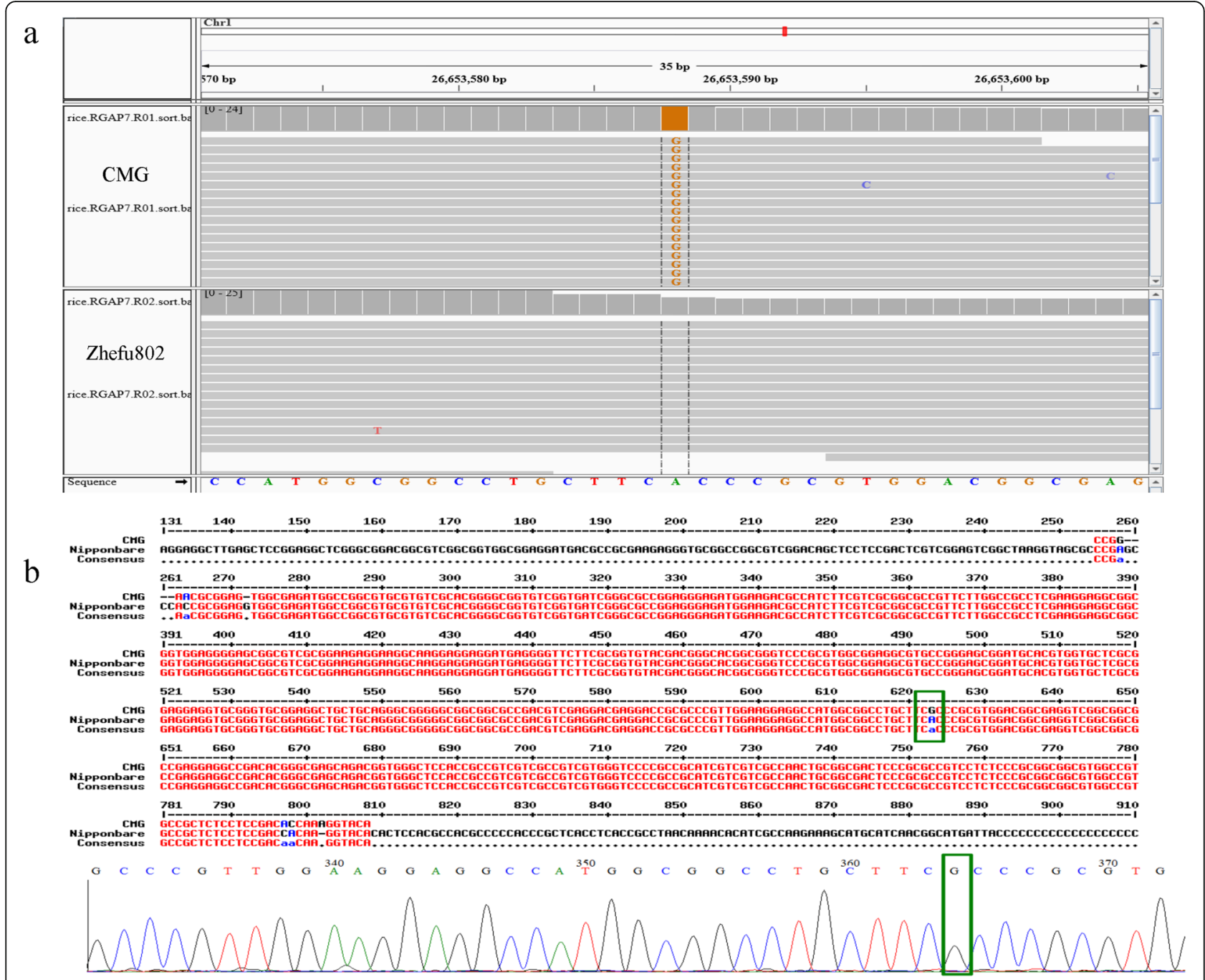

Fig. 6 Detection and validation of the candidate gene OSPP2C tolerant to salt at the seedling stage. a: The detected sequence variant of OsPP2C based on genome sequencing; $\mathbf{b}$ : sequencing and alignment of amplified PCR products using the specific primers for OsPP2C

Among 13 DEGs enriched in the ABA signal transduction pathway in the seedling stage, one (Os02g0766700), two (Os03g0297600 and Os05g0473101), and 10 genes belong to the ABF (ABRE binding factors), PYR/PYL, and PP2C families, respectively. Os02g0766700 encodes a bZIP transcription factor. Previous studies have indicated that bZIP transcription factors play crucial roles in the ABA signaling pathway in plants (Amir Hossain et al., 2010; Liu et al., 2014). The PYR/PYL family has been identified as ABA receptors (Liu et al., 2012; Ludwików, 2015). In the model of ABA signaling in plants, PP2Cs first interact with SnRK2s to form a reversible regulatory module in the manner of ABA-independence (Umezawa et al., 2009). The PYR-PP2C-SnRK2 complex is the primary framework for ABA signaling and phosphorylates downstream substrates, including bZIP transcription factors to activate ABA-responsive gene expression. We detected a sequence variant in the CDS region of OsPP2C8 from CMG, Zhefu802, and Nipponbare to alter the corresponding amino acid, which could cause the activation of the downstream genes in the pathway of ABA signaling to increase the salinity tolerance of CMG.

\section{Materials and Methods Materials}

A rice salinity tolerant landrace, Changmangu (CMG), was collected from a coastal beach in Zhanjiang city, Guangdong Province, China. Zhefu802 is a rice cultivar that is sensitive to salinity.

Approximately 2000 plants in the $\mathrm{F}_{2}$ population were derived from a cross between CMG and Zhefu802. Their 
leaves were harvested for BSA-seq to map the salinity tolerant genes. The seeds from the $F_{2}$ plants $\left(F_{2: 3}\right)$ were also collected to identify salinity tolerance. The seeds of more than $1000 \mathrm{~F}_{2: 3}$ lines from the $\mathrm{F}_{2}$ population and two parents were immersed in $120 \mathrm{mM} \mathrm{NaCl}$ solution for 4 days to investigate germination rate at $25^{\circ} \mathrm{C}$ in the artificial climate incubator (MGC-450HP). The $\mathrm{F}_{2: 3}$ lines with more than $40 \%$ germination rate continued to be cultivated under salt stress for 21 days. Thirty $\mathrm{F}_{2: 3}$ lines growing well were selected and their corresponding $F_{2}$ plant leaves were used to construct the salt tolerant bulk.

\section{Bulked Segregant Analysis by Pooled Sequencing}

BSA-seq was used to identify the genes regulating the tolerance to salinity in the above mentioned $F_{2}$ population. We selected 30 extremely tolerant and $30 \mathrm{~F}_{2}$ plants sensitive to salinity to create extreme sample pools. Genomic DNA was extracted using a modified CTAB (Hexadecyltrimethylammoniumbromide) method and purified by chloroform: phenol (1:1) (Chen and Ronald 1999). The DNA quality was checked using an Agilent bioanalyzer 1000 (Agilent Technologies, Singapore). Library preparation was performed according to the manufacturer's protocol. Genomic re-sequencing was conducted to generate paired-end 100-base (PE100) reads using the Illumina Hiseq 2000 platform (Illumina Technologies), which was conducted by Biomarker (China). Clean reads were aligned to reference genome sequences of the Japonica rice Nipponbare genome (http://ftp.ensemblgenomes.org/pub/release-24/plants/fasta/oryza_sativa/ dna/Oryza_sativa.IRGSP-1.0.24.dna.toplevel.fa.gz) using BWA software (Li and Durbin, 2009). SNPs and small InDelInDels were detected using GATK software (McKenna et al., 2010). The tool of Mark Duplicate in Picard (http://sourceforge.net/projects/picard/) was used to eliminate PCR duplication to increase SNP/InDel-calling accuracy. SNP/InDel-index was calculated for all the SNP/InDel positions. We excluded SNP/InDel positions with multiple genotypes and read depth $<4$ from the two bulk sequences. The association analysis was conducted by Euclidean Distance (ED) and SNP/InDelindex, respectively (Hill et al., 2013; Fekih et al., 2013). The overlapped regions based on the above two methods were considered candidate regions for salinity tolerance.

\section{Sample Preparation and Transcriptome Sequencing}

Dry seeds from CMG were immersed in $120 \mathrm{mMNaCl}$ solution and sterile water for 4 days. The young buds and roots were harvested at germination and rapidly stored in liquid nitrogen for transcriptomic sequencing. Thirty-day-old seedlings in nutrient solution were treated with $120 \mathrm{mM} \mathrm{NaCl}$ solution for $0 \mathrm{~min}, 30 \mathrm{~min}, 3$ $\mathrm{h}$, and $24 \mathrm{~h}$; approximately $2 \mathrm{~g}$ of roots per treatment were collected and rapidly stored in liquid nitrogen for transcriptomic sequencing of the seedling stage. Total RNA samples were extracted using the TRIzol reagent (Invitrogen) and then treated by RNase-freeDNase I (Takara) to remove genomic DNA. mRNA libraries were created according to the standardprotocols provided by Illumina. The mRNA quality including mRNA concentration and fragment size was tested using Qubit2.0 and Agilent 2100.mRNA was enriched using Dynabeads oligo (dT) (Dynal; Invitrogen) and fragmented using fragmentationbuffer. Double-stranded cDNAs were produced using reversetranscriptase (Superscript II; Invitrogen) and random hexamerprimers and further purified using AMPure XP beads. The purified double-stranded cDNA samples were enriched by PCR to construct the final cDNA libraries for sequencing using Hiseq 2500 (150 bp paired ends) by Biomarker (China). All raw-sequence reads data were uploaded to NCBI SequenceRead Archive (SRA, http://www.ncbi.nlm.nih.gov/Traces/sra) with accession numbers SRP143635.

Clean reads were also aligned toreference genome sequences of the Japonica rice Nipponbare genome using TopHat2 (Kim et al., 2013). Gene expression differences among different sampling points were detected using the EBSeq package (v1.10.1). Flod change $\geq 2$ and False Discovery Rate $(\mathrm{FDR})<0.01$ were set to act as the standard for screening the DEGs. Functional classification of DEGs, including Gene Ontology and KEGG pathways, were analyzed using the GOseq $\mathrm{R}$ package (Release2.12) and KOBAS software (v2.0).

\section{Real-Time PCR Confirmation of DEGs}

A total of 30 DEGs were randomly selected to confirm the transcriptomic sequencing results using real time PCR (RT-PCR). The corresponding sequences of these genes were obtained from the rice genome sequence database (Rap-db). These primers were designed according to the CDS sequences of the corresponding genes using Primer3 software (http://frodo.wi.mit.edu/) (Table S1). The Osactin1 gene was used as the internal control. Total RNAs were isolated from thesame samples for transcriptomic sequencing using the TRIzol reagent (Invitrogen) for RT-PCR. First-strand cDNA was synthesized from $1 \mathrm{mg}$ of DNase I-treated RNA samples in a $20 \mu \mathrm{l}$ reaction solution with the oligo $(\mathrm{dT})$ primer, using a Rever TraAce-akit (TOYOBO). Standard RT-qPCR was performed using SYBR Green SuperMix (Bio-Rad) on a CFX96 Real Time System (BioRad).

\section{Assays of abscisic Acid Level}

Thirty-day-old seedlings of Zhefu802 and CMG were treated in $120 \mathrm{mM} \mathrm{NaCl}$ solution for 0 min (mock), 30 min (SSI), $3 \mathrm{~h}$ (SSII), and $24 \mathrm{~h}$ (SSIII). Ten young seedlings were prepared at every sampling point to assay the 
level of ABA. The ABA content was scored using the plant abscisic acidELISA kits (AndyGene) according to the manufacturer's protocol.

\section{Conclusions}

In this study, we mapped six candidate regions for salt tolerance on chromosome 1 based on BSA-seq using extreme populations and identified 32 candidate genes according to the sequence polymorphism in the regions of promoters and ORFs between CMG and Zhefu802. The transcriptome analysis under the conditions of salt stress and normal growth identified numerous DEGs at the germination and young seedling stages. We investigated the expression of the 32 candidate genes and found that OsPP2C8 (Os01g0656200) was differentially expressed in the seedling stage under salt stress. Hence, OsPP2C8 was identified as the target candidate gene for salinity tolerance in the seedling stage, which will provide an important genetic resource for salt tolerant rice breeding.

\section{Supplementary information}

Supplementary information accompanies this paper at https://doi.org/10. 1186/s12284-019-0360-4

Additional file 1: Table S1. The mapped candidate regions based on SNP-index and ED. Table S2. The mapped candidate regions based on InDel-index and ED. Table S3. The overlapped candidate regions based on polymorphic SNPS and InDels. Table S4. Characteristics of the RT-PCR primers for validating the results of transcriptomic sequencing. Table S5. Functional annotation of the DEGs in four types of expression patterns at the seedling stage

\section{Abbreviations}

ABA: Abscisic acid; bHLH: Basic helix-loop-helix; BSA: Bulked segregant analysis; bZIP: Basic leucine zipper; DEG: Differentially expressed gene; ED: Euclidean distance; FDR: False discovery rate; GAMYB: GA-regulated myeloblastosis; NAC: NAM (no apical meristem), ATAF, CUC (cup-shaped cotyledon); NGS: Next generation sequencing; ORFs: Open reading frames; PP2C: Proteinphosphatase 2C; PYR1/PYL: Pyrabactin resistance 1/PYR1-like; RAP: RNA Polymerase II-associated Protein; SNP: Single nucleotide polymorphism;: TFs: Transcription factors

\section{Acknowledgments}

This work is supported in part by grants from Natural Science Foundation of Guangdong Province, China (2017A030310094), Science and Technology Program of Guangzhou, China (201707010218), Agricultural basic long-term scientific and technological work (ZX01S200101), Provincial Science and technology project of Guangdong (2014B070706013, 2016B070701011, 2017B030314173 and 2017A070702006).

\begin{abstract}
Authors' Contributions
Sun BR, screening the rice line, constructing the mapping population and designing the experiments; Fu CY, analyzing sequencing data and writing the manuscript; Fan $Z L$ and Chen Y, identifying the mapping population; Chen WF, Zhang J, Jiang LQ and LV SW, validating the partial sequencing results; Pan DJ and $\mathrm{Li} C$, organizing the relative experiments.
\end{abstract}

\section{Funding}

This work is supported in part by grants from Natural Science Foundation of Guangdong Province, China (2017A030310094), Science and Technology Program of Guangzhou, China (201707010218), Agricultural basic long-term scientific and technological work (ZX01S200101) and Provincial Science and technology project of Guangdong (2014B070706013, 2016B070701011, 2017B030314173 and 2017A070702006).

\section{Availability of Data and Materials}

All data generated or analysed during this study are included in this published article and its supplementary information files.

Ethics Approval and Consent to Participate

Not applicable.

\section{Consent for Publication}

Not applicable.

\section{Competing Interests}

The authors declare that they have no competing interests.

\section{Author details}

${ }^{1}$ Rice Research Institute, Guangdong Academy of Agricultural Sciences, Guangzhou 510640, China. ${ }^{2}$ Guangdong Provincial Key Laboratory of New Technology in Rice Breeding, Guangzhou, China.

Received: 5 January 2019 Accepted: 19 December 2019

Published online: 27 December 2019

\section{References}

Amir Hossain M, Lee Y, Cho Jl, Ahn CH, Lee SK, Jeon JS, Kang H, Lee CH, An G, Park PB (2010) The bZIP transcription factor OsABF1 is an ABA responsive element binding factor that enhances abiotic stress signaling in rice. Plant Mol Biol 72:557-566. https://doi.org/10.1007/s11103-009-9592-9

Ammar MHM, Pandit A, Singh RK, Sameena S, Chauhan MS, Singh AK, Sharma PC, Gaikwad K, Sharma TR, Mohapatra T, Singh NK (2009) Mapping of QTLS controlling $\mathrm{Na}^{+}, \mathrm{K}^{+}$and $\mathrm{Cl}^{-}$ion concentrations in salt tolerant indica rice variety CSR27. J Plant Biochem Biotechnol 18:139-150. https://doi.org/10. 1007/BF03263312

Bhatnagar N, Min MK, Choi EH, Kim N, Moon SJ, Yoon I, Kwon T, Ki-Hong Jung KH, Kim BG (2017) The protein phosphatase 2C clade a protein OsPP2C51 positively regulates seed germination by directly inactivating OsbZIP10. Plant Mol bio 193:389-401. https://doi.org/10.1007/s11103-016-0568-2

Chen DH, Ronald PC (1999) A rapid DNA minipreparation method suitable for AFLP and other PCR applications. Plant Mol Biol Rep 17:53-57. https://doi. org/10.1023/A:1007585532036

Cui MH, Yoo KS, Hyoung S, Nguyen HT, Kim YY, Kim HJ, Ok SH, Yoo SD, Shin JS (2013) An Arabidopsis R2R3-MYB transcription factor, AtMYB20, negatively regulates type $2 \mathrm{C}$ serine/threonine protein phosphatases to enhance salttolerance. FEBS Lett 587(12):1773-1778. https://doi.org/10.1016/j.febslet. 2013.04.028

Duan HK, Zhu Y, Qi DD, Li WL, Hua XJ, Liu YX, Deng X (2012) Comparative study on the expression of genes involved into carotenoid and ABA biosynthetic pathway in response to salt stress in tomato. J Integr Agr 11(7):1093-1102. https://doi.org/10.1016/s2095-3119(12)60102-6

Fekih R, Takagi H, Tamiru M, Abe A, Natsume S, Yaegashi H, Sharma S, Sharma S, Kanzaki H, Matsumura H, Saitoh H, Mitsuoka C, Utsushi H, Uemura A, Kanzaki E, Kosugi S, Yoshida K, Cano L, Kamoun S, Terauchi R (2013) MutMap+: genetic mapping and mutant identification without crossing in Rice. PLOS One 8(7):e68529. https://doi.org/10.1371/journal.pone.0068529

Gong JM, He P, Qian QA, Shen LS, Zhu LH, Chen SY (1999) Identification of salttolerance QTL in rice (Oryza sativa L.). Chin Sci Bull 44:68-71. https://doi.org/ 10.1007/BF03182889

Guo GJ, Wang SB, Liu JB, Pan BG, Diao WP, Ge W, Gao CZ, Snyder JC (2017) Rapid identification of QTLs underlying resistance to Cucumbermosaic virus in pepper (Capsicum frutescens). Theor Appl Genet 130:41-52. https://doi.org/10. 1007/s00122-016-2790-3

Hill JT, Demarest BL, Bisgrove BW, Gorsi B, Su YC, Yost HJ (2013) MMAPPR: mutation mapping analysis pipeline for pooled RNA-seq. Genome Res 23: 687-697. https://doi.org/10.1101/gr.146936.112

Huang X, Lu T, Han B (2013) Resequencing rice genomes: an emerging new era of rice genomics. Trends Genet 29:225-232. https://doi.org/10.1016/j.tig.2012. 12.001

Hwang SG, Chen HC, Huang WY, Chu YC, Shii CT, Cheng WH (2010) Ectopic expression of rice OSNCED3 in Arabidopsis increases ABA level andalters leaf morphology. Plant Sci 178:12-22. https://doi.org/10.1016/j.plantsci.2009.09.014 
Kim D, Pertea G, Trapnell C, Pimentel H, Kelley R, Salzberg SL (2013) TopHat2: accurate alignment of transcriptomes in the presence of insertions, deletions and gene fusions. Genome Biol 14:R36. https://doi. org/10.1186/gb-2013-14-4-r36

Koyama LM, Levesley A, Koebner RMD, Flowers TJ, Yeo AR (2001) Quantitative trait loci for component physiological traits determining salt tolerance in rice. Plant Physiol 125:406-422. https://doi.org/10.1104/pp.125.1.406

Kumar K, Kumar M, Kim SR, Ryu H, Cho YG (2013) Insights into genomics of salt stress response in rice. Rice 6(1):27. https://doi.org/10.1186/1939-8433-6-27

Kumar V, Singh A, Amitha Mithra SV, Krishnamurthy SL, Parida SK, Sourabh Jain S, Tiwari KK, Kumar P, Rao AR, Sharma SK, Khurana JP, Singh NK, Mohapatra T (2015) Genome-wide association mapping of salinity tolerance in rice (Oryza sativa). DNA Res 22(2):133-145. https://doi.org/10.1093/dnares/dsu046

Li H, Durbin R (2009) Fast and accurate short read alignment with burrowswheeler transform. Bioinformatics 25(14):1754-1760. https://doi.org/10.1093/ bioinformatics/btp324

Li ZK, Xu JL (2007) Breeding for drought and salt tolerant rice (oryza sativa L.): progress and perspectives. In: Jenks MA et al (eds) Advances in molecular breeding toward drought and salt tolerant crops. Springer, USA, pp 531-564

Lim JH, Yang HJ, Jung KH, Yoo SC, Paek NC (2014) Quantitative trait locus mapping and candidate gene analysis for plant architecture traits using whole genome re-sequencing in rice. Mol cells 37(2): 149-160. Doi:https://doi. org/10.14348/molcells.2014.2336

Lin HX, Zhu MZ, Yano M, Gao JP, Liang ZW, Su WA, Hu XH, Ren ZH, Chao DY (2004) QTLs for $\mathrm{Na}^{+}$and $\mathrm{K}^{+}$uptake of the shoots and roots controlling rice salt tolerance. Theor Appl Genet 108:253-260. https://doi.org/10.1007/ s00122-003-1421-y

Liu C, Mao B, Ou S, Wang W, Liu L, Wu Y, Chu C, Wang X (2014) OsbZIP71, a bZIP transcription factor, confers salinity and drought tolerance in rice. Plant Mol Bio I84:19-36. https://doi.org/10.1007/s11103-013-0115-3

Liu X, Zhu YM, Hong ZH, Cai H, Ji W, Luo X, Li J, Bai X (2012) AtPP2CG1, a protein phosphatase $2 \mathrm{C}$, positively regulates salt tolerance of Arabidopsis in abscisic acid-dependent manner. Biochem Biophys Res Commun 422:710-715. https://doi.org/10.1016/j.bbrc.2012.05.064

Ludwików A (2015) Targeting proteins for proteasomal degradation-a new function of Arabidopsis ABI1 protein phosphatase 2C. Front Plant Sci 6:310. https://doi.org/10.3389/fpls.2015.00310

Mahajan S, Tuteja N (2005) Cold, salinity and drought stresses: An overview. Arch Biochem Biophys 444(2):139-158. https://doi.org/10.1016/j.abb.2005.10.018

McKenna A, Hanna M, Banks E, Sivachenko A, Cibulskis K, Kernytsky A, Garimella K, Altshuler D, Gabriel S, Daly M, MA DP (2010) The Genome Analysis Toolkit: a MapReduce framework for analyzing next generation DNA sequencing data. Genome Res 20:1297-1303. https://doi.org/10.1101/gr.107524.110.20

Pandey MK, Khan AW, Singh VK, Vishwakarma MK, Shasidhar Y, Kumar V, Garg V, Bhat RS, Chitikineni A, Janila P, Guo BZ, Varshney RK (2017) QTL-seq approach identified genomic regions and diagnostic markers for rust and late leaf spot resistance in groundnut (Arachishypogaea L.). Plant Biotechnol J 15:927-941. https://doi.org/10.1111/pbi.12686

Pandit A, Rai V, Bal S, Sinha S, Kumar V, Chauhan M, GautamRK SR, Sharma PC, Singh AK, Gaikwad K, Sharma TR, MohapatraT SNK (2010) Combining QTL mapping and transcriptome profiling of bulked RILs for identification of functional polymorphism for salt tolerance genes in rice (Oryza sativa L.). Mol Gen Genomics 284:121-136. https://doi.org/10.1007/s00438-010-0551-6

Rahman MA, Thomson MJ, Shah-E-Alam M, Md O, Egdane J, Ismail AM (2016) Exploring novel genetic sources of salinity tolerance in rice through molecular and physiological characterization. Ann Bot-London 117:10831097. https://doi.org/10.1093/aob/mcw030

Ren ZH, Gao JP, Li LG, Cai XL, Huang W, Chao DY, Zhu MZ, Wang ZY, Luan S, Lin HX (2005) A rice quantitative trait locus for salt tolerance encodes a sodium transporter. Nat Genet 37:1141-1146. https://doi.org/10.1038/ng1643

Roy SJ, Tucker EJ, Tester M (2011) Genetic analysis of abiotic stress tolerance in crops. Curr Opin Plant Biol 14:232-239. https://doi.org/10.1016/j.pbi.2011.03.002

Takagi H, Tamiru M, Abe A, Yoshida K, Uemura A, Yaegashi H, Obara T, Oikawa K, Utsushi H, Kanzaki E, Mitsuoka C, Natsume S, Kosugi S, Kanzaki H, Matsumura H, Urasaki N, Kamoun S, Terauchi R (2015) MutMap accelerates breeding of a salt-tolerant rice cultivar. Nat biotechno 133: 445-449. https://doi.org/10.1038/ nbt.3188

Thomson MJ, de Ocampo M, Egdane J, Rahman MA, Sajise AG, Adorada DL, Tumimbang-Raiz E, Blumwald E, Seraj ZI, Singh RK, Gregorio GB, Ismail AM (2010) Characterizing the Saltol quantitative trait locus for salinity tolerance in rice. Rice 3:148-160. https://doi.org/10.1007/s12284-010-9053-8
Umezawa T, Sugiyama N, Mizoguchi M, Hayashi S, Myouga F, YamaguchiShinozaki K, Ishihama Y, Hirayama T, Shinozaki K (2009) Type 2C protein phosphatases directly regulate abscisic acid-activated protein kinases in Arabidopsis. Proc Natl Acad Sci U S A 106(41):17588-17593. https://doi.org/ 10.1073/pnas.0907095106

Varshney RK, Terauchi R, McCouch SR (2014) Harvesting the promising fruits of genomics: applying genome sequencing technologies to crop breeding. PLOS Biol 12(6):e1001883. https://doi.org/10.1371/journal.pbio.1001883

Walia H, Wilson C, Condamine P, Liu X, Ismail AM, Zeng L, Wanamaker SI, Mandal J, Xu J, Cui X, Close TJ (2005) Comparative transcriptional profiling of two contrasting rice genotypes under salinity stress during the vegetative growth stage. Plant Physiol 139(2):822-835. https://doi.org/10.2307/4281915

Wang Z, Chen Z, Cheng J, Lai Y, Wang J, Bao Y, Huang J, Zhang HS (2013) QTL analysis of $\mathrm{Na}^{+}$and $\mathrm{K}^{+}$concentrations in roots and shoots under different levels of $\mathrm{NaCl}$ stress in rice (Oryza sativa L.). PLoS One 7:e51202. https://doi. org/10.1371/journal.pone.0051202

Wong CE, Li Y, Labbe A, Guevara D, Nuin P, Whitty B, Diaz C, Golding GB, Gray GR, Weretilnyk EA, Griffith M, Moffatt BA (2006) Transcriptional profiling implicates novel interactions between abiotic stress and hormonal responses in Thellungiella, a close relative of Arabidopsis. Plant Physiol140(4):1437-1450. https://doi.org/10.2307/20205705

\section{Publisher's Note}

Springer Nature remains neutral with regard to jurisdictional claims in published maps and institutional affiliations.

\section{Submit your manuscript to a SpringerOpen ${ }^{\circ}$ journal and benefit from:}

- Convenient online submission

- Rigorous peer review

- Open access: articles freely available online

High visibility within the field

- Retaining the copyright to your article

Submit your next manuscript at $\boldsymbol{\nabla}$ springeropen.com 